\title{
Gene amplification in breast cancer - looking for the molecular mechanisms
}

\author{
Sergei Kovalenko \\ From 16th International Charles Heidelberger Symposium on Cancer Research \\ Coimbra, Portugal. 26-28 September 2010
}

Amplification of genes in breast cancer cells was shown to be coordinated in the majority of cases assuming the presence of common undisclosed mechanism underlying intrachromosomal gene amplification process.

Overexpression and amplification of the HER-2/neu gene is observed in $20-30 \%$ of breast cancer cases and predicts more frequent relapse and shorter survival time. However, the initial events and molecular mechanisms of chromosomal aberrations in the region around $H E R 2 / n e u$ as well as the precise boundaries of amplified regions remain to be obscure. This study was an attempt to define the boundaries of amplified chromosome 17q12-q21 region around HER2/neu gene. Structural peculiarities of amplification boundary regions were investigated by various techniques.

Gene dosage analysis was performed for several genes located around HER2/neu gene in 154 breast cancer tissue samples. The copy number of the ppparbp, her $2 / n e u$, znfn $1 a 3$ Casc 3 and top $2 a$ genes were analyzed by comparative TaqMan real time PCR. Fifty six samples (36 \%) were found to be her $2 /$ neu-amplified $\left(N_{H E R 2 / n e u} \geq 1,5\right)$, the boundaries for HER2-containing amplicons were mapped.

Observed results suggest that similar positions of amplified region boundaries in different breast cancer samples are not accidental and may be related with the DNA structural and/or sequence peculiarities of boundary regions. Trying to find "special" sequences at the amplicon boundaries, we performed the analysis of sequence flexibility in the region TBC1D3- HER2/neu $T O P 2 A$ using the TwistFlex program. The analysis revealed several high flexible sequences in SOCS7, FBX047, HER2/neu and ZNFN1A3 genes. The locations of amplified region boundaries in 30\% of cases were inside ZNFN1A3 gene. Organization of DNA sequence and nucleotide composition of FlexZNF-1 and FlexZNF-2 were similar to AT-rich islands of common fragile site (FS). Sequences of FlexZNF-1 and FlexZNF-2 have strong potential to form secondary structures according to the analysis by Mfold software (GCG Wisconsin Package ${ }^{\mathrm{TM}}$ ).

Thus, the analysis of DNA sequence in TBC1D3$H E R 2 / n e u$ - TOP $2 A$ region revealed the existence of two flexible sequences in the ZNFN1A3 gene sequence with the strong potential to form stable secondary structures which may affect normal DNA replication at these sites and result in DNA double strand break appearance. The location of FlexZNF-1 and FlexZNF-2 strongly coincided with the location of amplified around HER2/ neu gene fragment boundaries observed by real time PCR analysis.

This observation indicates involvement of Flex sites in the process of intrachromosomal DNA amplification.

Published: 24 September 2010

doi:

Cite this article as: Kovalenko: Gene amplification in breast cancer looking for the molecular mechanisms. BMC Proceedings 2010 4(Suppl 2): O20. 